\title{
Kinetic and thermodynamic study of bovine serum albumin interaction with rifampicin using surface plasmon resonance and molecular docking methods
}

Maryam Sharifi

Jafar Ezzati Nazhad Dolatabadi

Farzaneh Fathi

Mohammad Rashidi

Behzad Jafari

Habib Tajalli

Mohammad-Reza Rashidi 


\title{
Kinetic and thermodynamic study of bovine serum albumin interaction with rifampicin using surface plasmon resonance and molecular docking methods
}

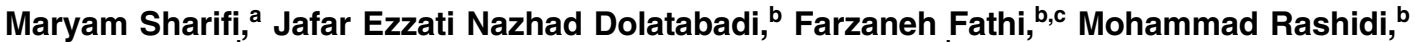 \\ Behzad Jafari, ${ }^{d}$ Habib Tajalli, ${ }^{a}$ and Mohammad-Reza Rashidi ${ }^{\mathrm{b}, *}$ \\ aUniversity of Tabriz, Research Institute for Applied Physics and Astronomy, Tabriz, Iran \\ ${ }^{b}$ Tabriz University of Medical Sciences, Research Center for Pharmaceutical Nanotechnology, Faculty of Pharmacy, Tabriz, Iran \\ 'Tabriz University of Medical Sciences, Student Research Committee, Tabriz, Iran \\ dTabriz University of Medical Sciences, Biotechnology Research Center, Tabriz, Iran
}

\begin{abstract}
The interaction of bovine serum albumin (BSA) with various drugs, such as antibiotics, due to the importance of BSA in drug delivery has attracted increasing research attention at present. Therefore, the aim of this study was investigation of BSA interaction with rifampicin using surface plasmon resonance (SPR) and molecular docking methods under the imitated physiological conditions $(\mathrm{pH}=7.4)$. BSA immobilization on carboxymethyl dextran hydrogel chip has been carried out after activation with $\mathrm{N}$-hydroxysuccinimide/ $\mathrm{N}$-ethyl-N-(3-diethylaminopropyl) carbodiimide. The dose-response sensorgrams of BSA upon increasing concentration of refampicin were attained in SPR analysis. The high affinity of rifampicin to BSA was demonstrated by a low equilibrium constants $\left(K_{D}\right)$ value $\left(3.46 \times 10^{-5}\right.$ at $\left.40^{\circ} \mathrm{C}\right)$. The process of kinetic values changing shows that affinity of BSA to rifampicin decreased with rising temperature. The positive value of both enthalpy change $(\Delta H)$ and entropy change $(\Delta S)$ showed that hydrophobic force plays major role in the BSA interaction with rifampicin. The positive value of $\Delta G$ was indicative of nonspontaneous and enthalpy-driven binding process. In addition, according to the molecular docking study, hydrogen binding has some contributions in the interaction of rifampicin with BSA. ๑ 2017 Society of Photo-Optical Instrumentation Engineers (SPIE) [DOI: 10.1117/1.JBO.22.3.037002]
\end{abstract}

Keywords: surface plasmon resonance; rifampicin; hydrophobic.

Paper 160775RR received Nov. 15, 2016; accepted for publication Feb. 10, 2017; published online Mar. 2, 2017.

\section{Introduction}

Serum albumin is the most abundant protein in the blood circulation and is well-known as transport protein. The most important function of albumins is transportation of various organic and inorganic compounds including drugs. ${ }^{1-4}$ Serum albumin interaction with various drugs plays a major role in estimation of the small molecules distribution, elimination, and transport in biological systems. Being familiar with the molecular basis of drug-albumin binding is crucial in designing new therapeutic agents for improvement of drug activity. Therefore, albumindrug binding study is important and may influence properties of typical drugs. Due to low cost, medical care, availability, and binding properties, bovine serum albumin (BSA) usually is selected for investigation of drug binding capability. ${ }^{5-7}$

Rifampicin (Fig. 1) is an example of antibiotics used for treatment of pulmonary tuberculosis. ${ }^{8,9}$ Despite the broad pharmacological application of rifampicin, its mechanism of action and effects on serum albumin is poorly understood and investigated using surface plasmon resonance (SPR). Therefore, study of BSA interaction with rifampicin could be a challenging issue and the development of a reliable analytical method, such as SPR that allows the measurement of rifampicin-albumin interaction kinetics, would be of great value. *Address all correspondence to: Mohammad-Reza Rashidi, E-mail: rashidi@
tbzmed.ac.ir
SPR is a sensitive label-free technique that detects the changes in refractive index at surfaces of metals. It can be used for study of biomolecular interactions with various drugs and ligands with high sensitivity. ${ }^{10-12}$ Label free, real-time and both qualitative and quantitative detection are examples of SPR advantages compared to other techniques. In addition, determination of kinetic constants and thermodynamic parameters is possible using this technique. ${ }^{12-14}$

To the best of our knowledge, interaction of rifampicin with BSA has not been reported using the SPR technique. Owing to the extensive application of rifampicin, investigation on BSA interaction needs to be addressed. Therefore, in the current study, we investigated the binding properties of rifampicin with BSA using SPR method and molecular docking analysis.

\section{Materials and Methods}

\subsection{Materials}

Rifampicin was purchased from Sigma-Aldrich (Steinheim, Germany). Phosphate-buffered saline (PBS) solution, acetate buffer, BSA, ethanolamine-HCl, N-hydroxysuccinimide (NHS), and N-ethyl-N-(3-diethylaminopropyl) carbodiimide (EDC) were purchased from Merck (Darmstadt, Germany). Carboxymethyl dextran (CMD) low-density-modified gold surfaces chip was prepared from Bionavis Company (Tampere-region, Finland).

$1083-3668 / 2017 / \$ 25.00$ (C) 2017 SPIE 


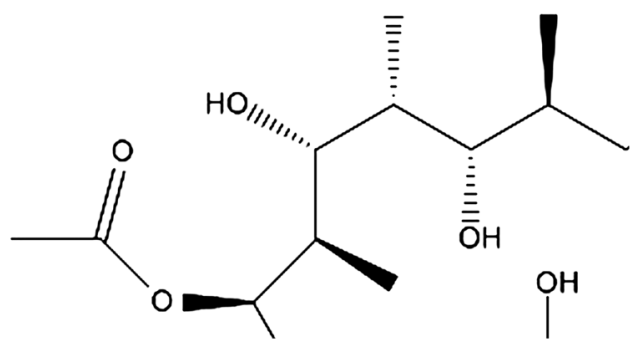

Fig. 1 Chemical structure of rifampicin.

\subsection{Apparatus}

All kinetic parameters were recorded with dual-flow channels SPR device (MP-SPR Navi 210A, BioNavis Ltd., Tampereregion, Finland).

\subsection{Bovine Serum Albumin Immobilization}

After putting a CMD sensor chip on SPR instrument, the whole device is rinsed with acetate buffer. As soon as reaching steady baseline in sensorgram, the chip surface cleaned with $\mathrm{NaCl}(2$ $\mathrm{M})$ and $\mathrm{NaOH}(0.1 \mathrm{M})$ by injecting them to both channels for 3 min. To activate the chip surface EDC: NHS 1:1 (NHS $0.05 \mathrm{M}$ + EDC $0.2 \mathrm{M}$ ) solution injected to the sensor chip for $7 \mathrm{~min}$. For immobilization of BSA, the BSA solution injected $(0.3 \mathrm{mg} / \mathrm{ml})$ to channel 1 , for $10 \mathrm{~min}$ and acetate buffer injected (running buffer) to channel 2 (reference channel). Finally, ethanolamine$\mathrm{HCl}(1 \mathrm{M}, \mathrm{pH} 8.5)$ was injected for blocking the remaining activated surface groups. All of the injection processes were performed with flow rate of $30 \mu \mathrm{l} / \mathrm{min}$.

\subsection{Kinetic Parameters of Bovine Serum Albumin Interaction with Rifampicin}

Rifampicin solution in PBS buffer at $\mathrm{pH} 7.4$ was injected using a flow rate of $30 \mu \mathrm{l} / \mathrm{min}$ for $3 \mathrm{~min}$ at various concentrations (1 to $128 \mu \mathrm{M}$ ). Both flow cells were used for sample injection and due to BSA immobilization at one flow cells, the flow cell without any BSA was used as reference. Rapid dissociation of rifampicin from BSA surface omits the need for regeneration process.

To estimate the affinity and kinetic of the BSA interaction with rifampicin, Trace Drawer ${ }^{\mathrm{TM}}$ for SPR $\mathrm{Navi}^{\mathrm{TM}}$ was used. Before calculations in Trace Drawer, data are extracted with SPR Navi ${ }^{\mathrm{TM}}$ Data viewer software.

\subsection{Thermodynamic Analysis}

The effect of temperature on binding of rifampicin to BSA was studied to estimate the thermodynamic parameters of rifampicin-BSA complex formation. Therefore, SPR measurements were investigated at four different temperatures $(298,303,308$, and $313 \mathrm{~K})$.

\subsection{Molecular Docking Simulation}

The docking study of BSA with rifampicin was performed by the open-source windows version of Auto Dock Vina 1.1.2 software. Auto Dock Vina not only improves the accuracy of molecular docking between the ligand and receptor but also increases the speed of dock running. ${ }^{15}$ For study of the molecular interaction of BSA with rifampicin by in silico simulation,

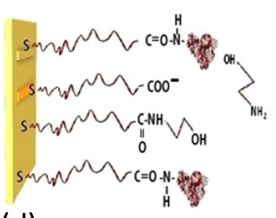

(d)

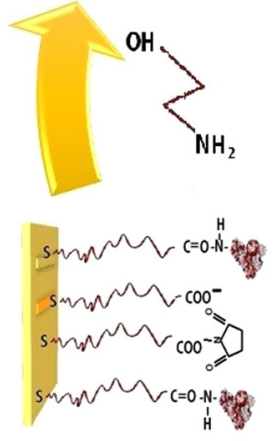

(c)

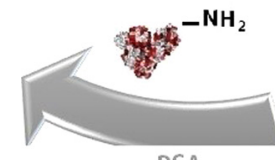

BSA

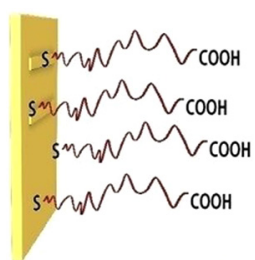

(a)

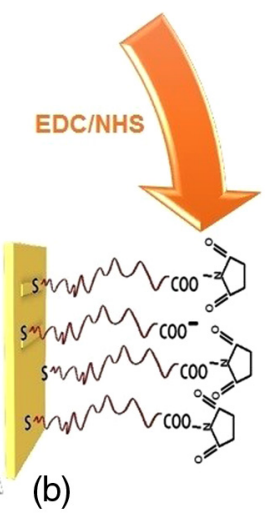

Fig. 2 Schematic illustration of BSA immobilization by amine coupling: (a) carboxymethyl dextran sensor chip, (b) activation of $\mathrm{COOH}$ in CMD via EDC/NHS before BSA immobilization, (c) immobilization of the BSA through covalent amide bond formation, and (d) deactivation of the remaining activated surface groups by blocking reagents ethanolamine, which is the most commonly used agent.

the x-ray structure of BSA (PDB ID code4F5S, $2.47 \AA$ resolutions) was selected from the Research Collaboratory for Structural Bioinformatics protein data bank. The rifampicin molecular structure was prepared by drawing the twodimensional chemical structure by Chem Draw Ultra 10.0 software and then for molecular energy optimization, the HyperChem 8.0.8 software was selected. Molecular structure optimization of rifampicin was done in two steps: at first the $\mathrm{MM}+$ molecular mechanics was performed and after completed this step the AM1 semiempirical procedures was executed. To minimize rifampicin structure, the Fletcher-Reeves algorithm was selected in both optimization processes. ${ }^{16}$ For the docking study, the extracrystallographic structure, such as water molecules, was removed from the BSA structure. The BSA structure and compound were changed by adding the polar hydrogens. Also, the Kollman charges as well as Gasteiger charges were added to the receptor and ligand, respectively. After attaining the pdbqt files of BSA and rifampicin, drug was inputted in determined six different binding sites of BSA. The grid box dimension size at grid points in $x \times y \times z$ directions were set to $27 \AA$ with a grid spacing of $1 \AA$ in Vina docking. The energy range and exhaustiveness value were set to $4 \mathrm{kcal} / \mathrm{mol}$ and 40 , respectively.

\section{Results and Discussion}

\subsection{Bovine Serum Albumin Immobilization}

Figure 2 shows the schematic illustration of BSA immobilization on the Au chip surface.

The initial curve of the aforementioned process before and after BSA immobilization was shown in Fig. 3. The blue curve shows channel 1 , in which BSA has been immobilized and showed angle shift around $0.98 \mathrm{deg}$. The resonance curves shift to higher incident angles and become wider due to new 

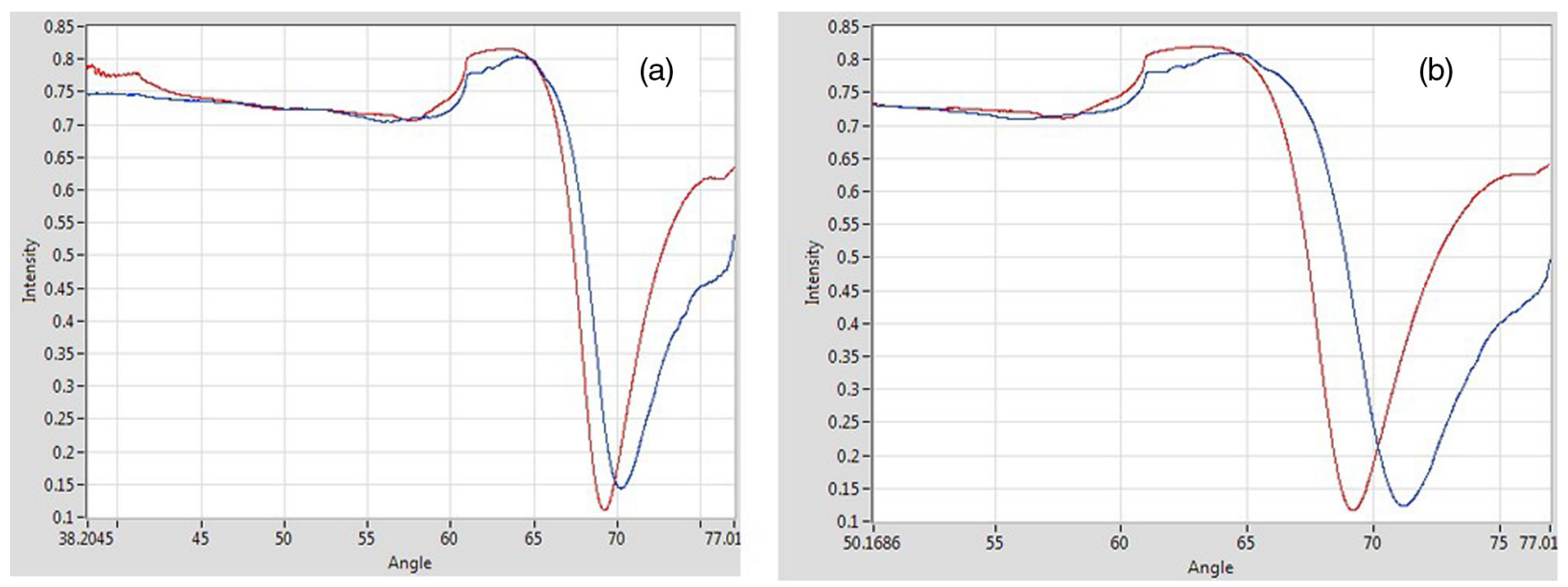

Fig. 3 Initial SPR curve (a) before and (b) after BSA immobilization.
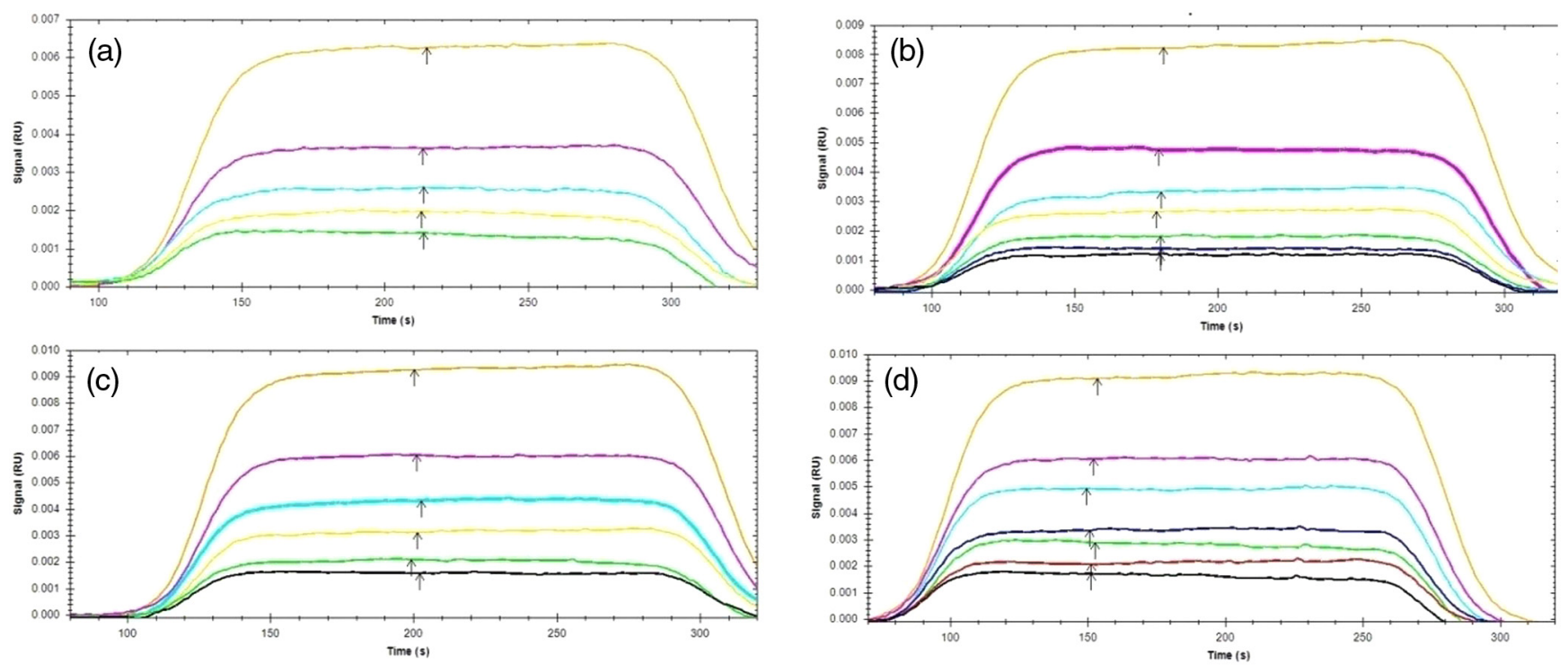

Fig. 4 Sensorgram of rifampicin interaction with immobilized BSA at various concentration 1 to $128 \mu \mathrm{M}$ at different temperatures (a) 298, (b) 303, (c) 308, and (d) $313 \mathrm{~K}$.

layer formation on Au sensor slide and increase in the thickness of the adsorbed layer on the surface. From the aforementioned results, it can be concluded that the BSA attachment was performed properly on the Au sensor slide through covalent amide bond formation. ${ }^{17}$

\subsection{Kinetics Binding of Bovine Serum Albumin and Rifamipcin}

The interaction between the analyte $(A)$ and the immobilized BSA $(B)$ can be demonstrated through simple Langmuirian mode of binding for complex formation $(A B)$. For reversible associations and dissociations in a chemical equilibrium, it can be defined by the following equation: ${ }^{17}$

$A+B \underset{K_{\mathrm{d}}}{\stackrel{K_{\mathrm{a}}}{\rightleftarrows}} A B$

where $k_{\mathrm{a}}$ is the association rate constant and $k_{\mathrm{d}}$ is the dissociation rate constant. To attain the kinetic features, calculation of the $k_{\mathrm{a}}$ and $k_{\mathrm{d}}$ rate constants and the equilibrium constants $\left(K_{D}\right)$ for the bindings of BSA to rifampicin is required. ${ }^{17,18}$ The $K_{D}$ is a measure of ligand affinity to various biomacromolecules, such as BSA and defined as $K_{D}=k_{\mathrm{d}} / k_{\mathrm{a}}$. Figure 4 shows the SPR sensorgrams of the reaction between BSA and rifampicin after reference subtraction at concentrations ranging from 1 to $128 \mu \mathrm{M}$ at four temperatures $(298,303,308$, and $313 \mathrm{~K})$.

Table 1 shows values of kinetic parameters at four temperatures. According to the Table $1, k_{\mathrm{a}}$ and $k_{\mathrm{d}}$ of BSA interaction with rifampicin decreased regularly upon rising temperature. It shows that high temperature led in the reaction rate decrease. The mean $K_{D}$ for rifampicin binding increased almost 2.30 from 15 to $34.6 \mu \mathrm{M}$ over this temperature range. The temperature dependence of the $K_{D}$ was due to an increase in the $k_{\mathrm{d}}$ value rather than the $k_{\mathrm{a}}$ value. About 15 -fold increase in the $k_{\mathrm{d}}$ value was observed when temperature increased from 298 to $313 \mathrm{~K}$. However, there was only sixfold increase in the $k_{\mathrm{a}}$ value when temperature rose to $313 \mathrm{~K}$. As it is clear in Table $1, K_{D}$ of BSA interaction with rifampicin increased upon rising temperature, 
Table 1 Association rate constant $\left(k_{\mathrm{a}}\right)$, dissociation rate constant $\left(k_{\mathrm{d}}\right)$, and equilibrium constants $\left(k_{D}\right)$ values of rifampicin interaction with BSA.

\begin{tabular}{lccc} 
Temperatures $(\mathrm{K})$ & $k_{\mathrm{a}}(1 / \mathrm{M} \times \mathrm{s})$ & $k_{\mathrm{d}}(1 / \mathrm{s})$ & $K_{D}(\mathrm{M})$ \\
\hline 298 & $1.48 \times 10^{2}$ & $2.22 \times 10^{-3}$ & $1.50 \times 10^{-5}$ \\
303 & $7.70 \times 10^{2}$ & $1.46 \times 10^{-2}$ & $1.90 \times 10^{-5}$ \\
308 & $6.83 \times 10^{2}$ & $1.71 \times 10^{-2}$ & $2.50 \times 10^{-5}$ \\
313 & $9.07 \times 10^{2}$ & $3.14 \times 10^{-2}$ & $3.46 \times 10^{-5}$ \\
\hline
\end{tabular}

which showed that affinity between two materials decreases upon rising temperatures.

\subsection{Thermodynamic Parameters Analysis of Binding Bovine Serum Albumin with Rifampicin}

To investigate the effect of temperature and thermodynamic features, the reaction between BSA and rifampicin was carried out at different temperatures. The type of interaction between BSA protein and rifampicin may consist of the following forces: hydrogen bond, Van der Waals, electrostatic, and hydrophobic forces. The value of thermodynamic parameters, such as enthalpy change $(\Delta H)$ and entropy change $(\Delta S)$, is used for the estimation of interaction types. The positive value of $\Delta H$ and $\Delta S$ shows that the hydrophobic forces play major roles in the binding of two molecules; the negative values represent
Van der Waals interactions and hydrogen bonds occurring and finally, the negative value of $\Delta H$ and positive amount of $\Delta S$ is indicative of electrostatic interaction happening. These parameters can be calculated through the van't Hoff Eq. (1) by the plot of $\ln K_{D}$ versus $1 / T^{19}$

$$
\ln K_{D}=-\frac{\Delta H}{R T}+\frac{\Delta S}{R},
$$

where $R$ and $T$ are the universal gas constant and temperature, respectively.

BSA binding study was performed at four different temperatures $(298,303,308$, and $313 \mathrm{~K})$. Figure 5 shows the plot of $\ln$ $K_{D}$ versus $1 / T$.

The total energy released by the reaction is calculated with the Gibbs-Helmholtz

$$
\Delta G=\Delta H-T \Delta S
$$

The positive values have been attained for both $\Delta H$ and $\Delta S$ according to Table 2 for the binding of BSA to rifampicin, which indicates that the binding processes are mainly enthalpydriven and the rifampicin-BSA interaction can be explained in terms of hydrophobic forces. Although Yu et $\mathrm{al}^{7}$ showed that electrostatic interactions play a major role in the BSA binding to rifampicin using fluorescence spectroscopy; according to some other authors, ${ }^{6}$ the interaction between rifampicin and BSA occurs through hydrophobic binding processes. Taking into account the lipophilicity of rifampicin, the involvement of the latter forces in the binding process of this drug with BSA

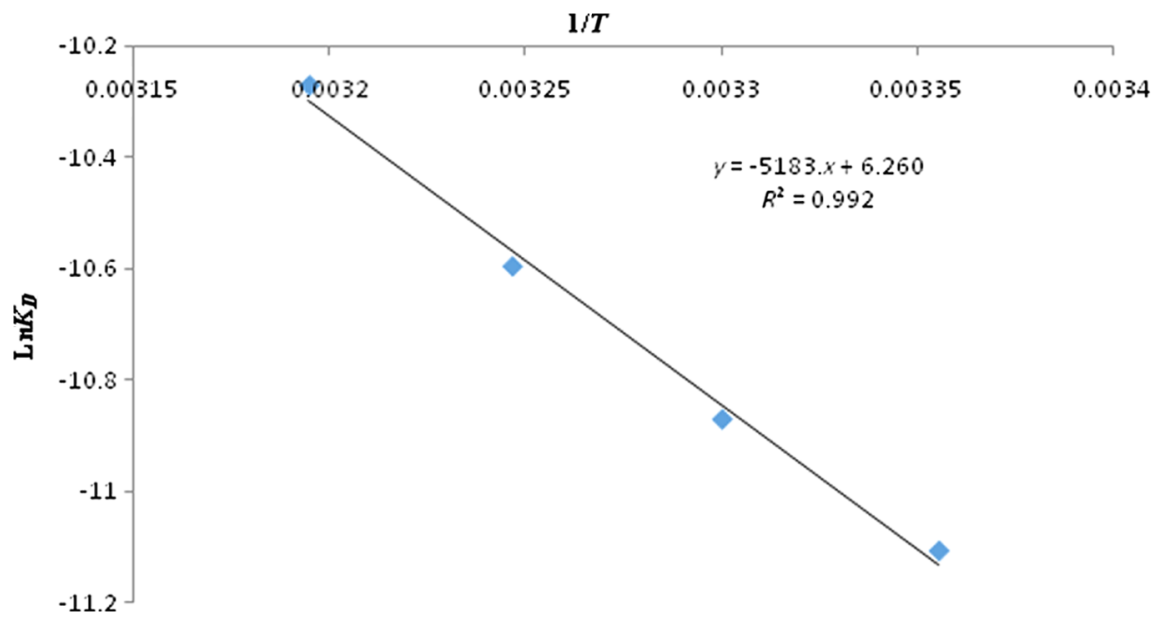

Fig. 5 Van't Hoff plot for the interaction of BSA and rifampicin.

\begin{tabular}{|c|c|c|c|c|}
\hline Temperatures $(\mathrm{K})$ & $K_{D}$ & $\Delta H\left(\mathrm{~kJ} \mathrm{~mol}^{-1}\right)$ & $\Delta S\left(\mathrm{~J} \mathrm{~mol}^{-1}\right)$ & $\Delta G\left(\mathrm{~kJ} \mathrm{~mol}^{-1} \mathrm{k}^{-1}\right)$ \\
\hline 298 & $1.50 \times 10^{-5}$ & & & 27.58735684 \\
\hline 303 & $1.90 \times 10^{-5}$ & 43.10066 & 52.05806822 & 27.3270665 \\
\hline 308 & $2.50 \times 10^{-5}$ & & & 27.06677616 \\
\hline 313 & $3.46 \times 10^{-5}$ & & & 26.80648582 \\
\hline
\end{tabular}

Table 2 Thermodynamic parameters of BSA binding with rifampicin at four different temperatures $(298,303,308$, and $313 \mathrm{~K})$. 
Table 3 The lowest binding free energy values $(\Delta G)$ calculated by Auto Dock Vina from rifampicin docking with six different binding sites of BSA.

\begin{tabular}{lcccccc}
\hline Binding site & Site IA & Site IIA & Site IIIA & Site IB & Site IIB & Site IIIB \\
\hline$E_{\text {lowest }}(\mathrm{kcal} / \mathrm{mol})$ & -4.3 & -6.0 & -4.2 & -6.5 & -7.0 & -6.5 \\
\hline
\end{tabular}

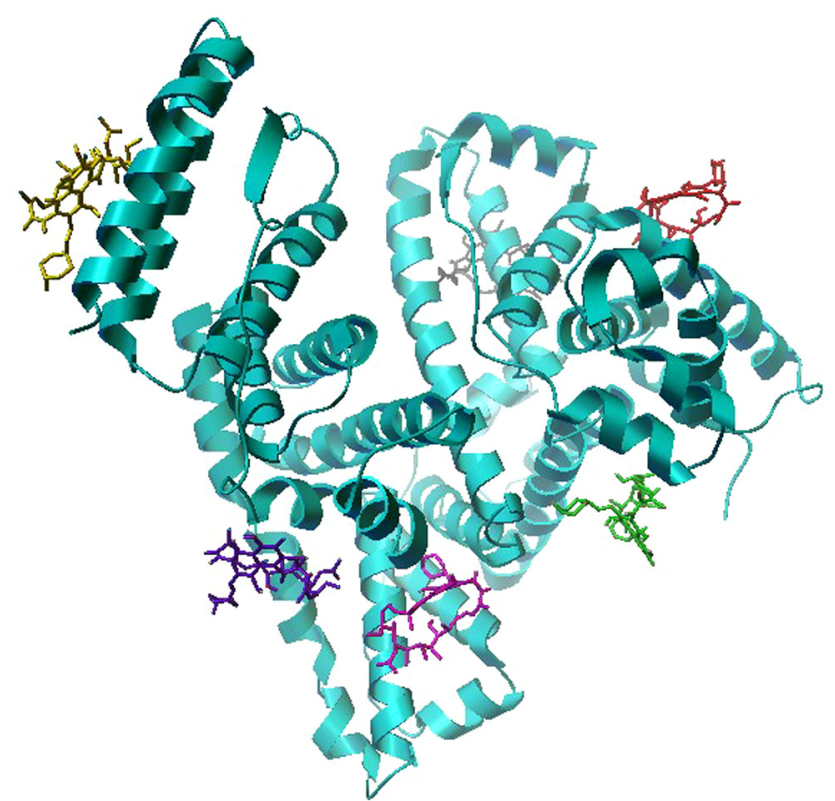

Fig. 6 The best docked conformation poses of rifampicin in six various binding sites of BSA. seems to be more logical. In addition, the positive value of $\Delta G$ means that the binding process was nonspontaneous and enthalpy driven and the formation of rifampicin-BSA complex was an endothermic process. ${ }^{2,20,21}$

\subsection{Molecular Docking Study}

Twenty mode runs were defined in our docking procedure. After molecular docking process, the best conformation of each rifampicin-BSA complexes relative to the lowest free binding energy $(\Delta G)$ according to the Vina cluster was selected and summarized in Table 3 and the lowest free binding energy conformer of docking for each binding site has been shown in Fig. 6 . The lowest free binding energy $(\Delta G)$ for the sites $A$ and $B$ of albumin molecule was found and presented in Table 3. The subdomain IIB and IIA of albumin molecule has some amino acids with functional groups that can make hydrogen binding as well (Fig. 7). Our docking analysis revealed some H-bondings between rifampicin and BSA (Table 4). Rifampicin has also several functional groups, such as hydroxyl with hydrogen binding capability. Therefore, the possibility of some hydrogen bindings between rifampicin and BSA cannot be totally excluded, although based on the results obtained from thermodynamic studies, this is the hydrophobic forces that act as the
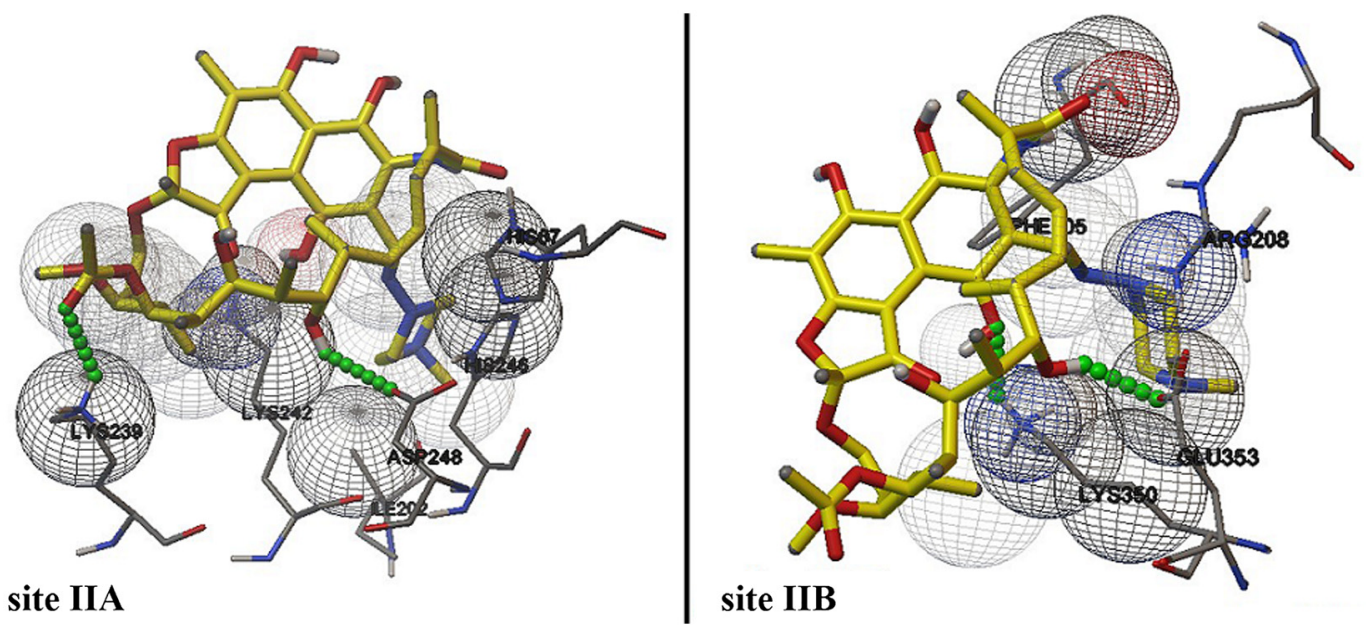

Fig. $7 \mathrm{H}$-bonding between rifampicin and different amino acids in two binding subdomains of BSA. Green spheres are $\mathrm{H}$-bonding and wireframe spheres are close contact between rifampicin and residues.

Table 4 Amino acids that contacted with rifampicin from the analyzing of all runs of each docking procedure and the number and type of amino acids that join together in formation of $\mathrm{H}$-bond in selected binding site of BSA (IIA and IIB). The number of phrases indicated the repeating of $\mathrm{H}$ bonding with dependent amino acid.

\begin{tabular}{llll}
$\begin{array}{l}\text { Binding } \\
\text { site }\end{array}$ & \multicolumn{1}{c}{ Amino acids that contacted with rifamipcin } & $\begin{array}{c}\text { Amino acids company in } \\
\text { H-bonding formation }\end{array}$ & Number of H-bonding \\
\hline Site IIA & $\begin{array}{l}\text { Arg10-His67-Ile202-Glu207-Arg208-Lys211-Lys232-Thr235-Lys239- } \\
\text { Lys242-Glu243-His246-Asp248-Asp255-Asp258-Lys261-Tyr262 }\end{array}$ & $\begin{array}{l}\text { Glu207-Lys211-Lys232- } \\
\text { Lys239-Lys242(6)-Asp248 }\end{array}$ & 11 \\
Site IIB & $\begin{array}{l}\text { Phe205-Arg208-Ala209-Lys322-Lys350-Glu351-Glu353-Ala354- } \\
\text { Glu357-Glu358-Lys362-Thr371-Lys375-His378-Leu379-Glu382-Asn482 }\end{array}$ & $\begin{array}{l}\text { Lys131(2)-Lys159(5)- } \\
\text { Asn161-Lys180-Lys187(5) }\end{array}$ \\
\hline
\end{tabular}


major driven forces in the interaction of rifampicin with the protein. Therefore, it seems that rifampicin interacts with BSA through hydrophobic interactions as the major driven forces. ${ }^{1,21}$

\section{Conclusion}

In this paper, we studied the interaction between BSA and rifampicin using SPR method and molecular docking analysis. The experimental results revealed that BSA has high affinity to rifampicin, which confirmed with low value of $K_{D}$. However, $K_{D}$ of BSA interaction with rifampicin increased upon rising temperature, which showed that affinity between two material decreases upon rising temperatures. The results of thermodynamic parameters showed that the main force between rifampicin and BSA is hydrophobic due to the hydrophobic nature of drug. Also positive value of $\Delta G$ confirmed that the binding between BSA and rifampicin is enthalpy-driven and nonspontaneous. In addition, molecular docking study showed the involvement of hydrogen binding in the formation of rifampicinBSA complex.

\section{Disclosures}

The authors declare no competing interests.

\section{Acknowledgments}

The authors are grateful for financial support from the Student Research Committee and Research Center for Pharmaceutical Nanotechnology, Tabriz University of Medical Sciences.

\section{References}

1. D. Agudelo et al., "Probing the binding sites of antibiotic drugs doxorubicin and $\mathrm{N}$-(trifluoroacetyl) doxorubicin with human and bovine serum albumins," PLoS One 7(8), e43814 (2012).

2. A. Belatik et al., "Binding sites of retinol and retinoic acid with serum albumins," Eur. J. Med. Chem. 48, 114-123 (2012).

3. A. Belatik et al., "Locating the binding sites of $\mathrm{Pb}(\mathrm{II})$ ion with human and bovine serum albumins," PLoS One 7(5), e36723 (2012).

4. J. S. Mandeville and H. A. Tajmir-Riahi, "Complexes of dendrimers with bovine serum albumin," Biomacromolecules 11(2), 465-472 (2010).

5. J. Flarakos, K. L. Morand, and P. Vouros, "High-throughput solutionbased medicinal library screening against human serum albumin," Anal. Chem. 77(5), 1345-1353 (2005).
6. B. P. Kamat and J. Seetharamappa, "Mechanism of interaction of vincristine sulphate and rifampicin with bovine serum albumin: a spectroscopic study," J. Chem. Sci. 117(6), 649-655 (2005).

7. O.-Y. Yu et al., "Probing the binding of rifampicin to bovine serum albumin in aqueous solution," J. Solution Chem. 40(10), 1711-1723 (2011).

8. M. S. Benedetti and P. Dostert, "Induction and autoinduction properties of rifamycin derivatives: a review of animal and human studies," Environ. Health Perspect. 102(Suppl. 9), 101-105 (1994).

9. A. Tupin et al., "Resistance to rifampicin: at the crossroads between ecological, genomic and medical concerns," Int. J. Antimicrob. Agents 35(6), 519-523 (2010).

10. R. J. Green et al., "Surface plasmon resonance analysis of dynamic biological interactions with biomaterials," Biomaterials 21(18), 1823-1835 (2000).

11. R. L. Rich and D. G. Myszka, "Advances in surface plasmon resonance biosensor analysis," Curr. Opin. Biotechnol. 11(1), 54-61 (2000).

12. J. Homola, "Surface plasmon resonance sensors for detection of chemical and biological species," Chem. Rev. 108(2), 462-493 (2008).

13. D. G. Myszka and R. L. Rich, "Implementing surface plasmon resonance biosensors in drug discovery," Pharm. Sci. Technol. Today 3(9), 310-317 (2000).

14. J. M. McDonnell, "Surface plasmon resonance: towards an understanding of the mechanisms of biological molecular recognition," Curr. Opin. Chem. Biol. 5(5), 572-577 (2001).

15. O. Trott and A. J. Olson, "AutoDock Vina: improving the speed and accuracy of docking with a new scoring function, efficient optimization and multithreading," J. Comput. Chem. 31(2), 455-461 (2010).

16. J. J. P. Stewart, "Optimization of parameters for semiempirical methods I. Method," J. Comput. Chem. 10(2), 209-220 (1989).

17. F. Fathi et al., "Kinetic studies of bovine serum albumin interaction with PG and TBHQ using surface plasmon resonance," Int. J. Biol. Macromol. 91, 1045-1050 (2016).

18. M. Ehteshami et al., "Characterization of 6-mercaptopurine binding to bovine serum albumin and its displacement from the binding sites by quercetin and rutin," J. Lumin. 135, 164-169 (2013).

19. Y. S. N. Day et al., "Direct comparison of binding equilibrium, thermodynamic, and rate constants determined by surface- and solution-based biophysical methods," Protein Sci.: Publ. Protein Soc. 11(5), 10171025 (2002).

20. F. Rasoulzadeh et al., "Fluorescence quenching study of quercetin interaction with bovine milk xanthine oxidase," Spectrochim. Acta, Part A 72(1), 190-193 (2009).

21. J. E. N. Dolatabadi et al., "Spectroscopic and molecular modeling studies of human serum albumin interaction with propyl gallate," RSC Adv. 4(110), 64559-64564 (2014).

Biographies for the authors are not available. 